\title{
Il Cibo Nella Letteratura di Primo Levi e nel Cinema di Roberto Benigni
}

Maria Franca Zuccarello ${ }^{1}$

ABSTRACT: La cucina italiana è il risultato di tradizioni, molte delle quali risalgono a tempi molto antichi ed ai molti popoli stranieri che hanno dominato le regioni italiane, apportandovi cultura ed innumerevoli ricette che ne fanno, nelle sue diversità, il prodotto Made in Italy, conosciuto in tutto il mondo. Molti autori hanno parlato del cibo in momenti di azione dei personaggi, attorno ad imbandite tavole di gente ricca e potente, o del campo, senza risorse per fare delle buone ricette. Però non parleremo qui dei piaceri del buon cibo italiano, ma della necessità del cibo come alimento che dà la forza per continuare a vivere. Ed allora, così come Primo Levi in Se 
questo è un uomo, ci domandiamo: è uomo chi mangia un cibo senza sapore, senza colore, senza odore e quindi senza piacere, che gli serve solo per sopravvivere? E vedremo anche la maniera giocherellona ed allegra, che all'inizio del film La vita è bella Benigni usa per parlare del cibo, mentre nella seconda parte, quando la sua vita non è più un gioco - malgrado lui la conduca come tale affinché il figlio non capisca dove sono stati portati, fa di tutto affinché non manchi al piccolo Giosuè.

PAROLE CHIAVE: letteratura; cinema; campi di concentramento; cibo.

RESUMO: A cozinha italiana é o resultado de tradições, muitas das quais remontam a tempos muito antigos e aos diversos povos estrangeiros que dominaram as regiões italianas, trazendo consigo cultura e inúmeras receitas que formam, em sua diversidade, o produto Made in Italy, conhecido em todo o mundo. Muitos autores falaram da comida, em momentos de ação dos personagens, ao redor de mesas fartas de gente rica e poderosa ou mesas pobres do campo, de gente sem recursos para fazer boas receitas. Mas nós não falaremos aqui dos prazeres da boa comida italiana, mas da necessidade da comida como alimento que dá forças para continuar a viver. E então, assim como Primo Levi em Se questo è un uomo,, nos perguntamos: é um homem quem come uma comida sem sabor, sem cor, sem cheiro e, portanto, sem prazer, que serve somente para a sobrevivência? E veremos também a maneira brincalhona e alegre que no início do filme $L a$ vita è bella, Benigni usa para falar da comida, enquanto na segunda parte, quando sua vida não é mais um jogo - embora ele a conduza como tal para que o filho não perceba para onde foram levados - faz de tudo para que nada falte ao pequeno Giosuè.

PALAVRAS CHAVE: literatura; cinema; campos de concentração; comida.

ABSTRACT: The Italian kitchen results from ancient traditions as well as from the innumerous foreigner people that had dominated the Italian regions to where they brought their culture and recipes of their own kitchen 
making the products made in Italy well known in the whole world. Several authors had talked about food and recipes during the characters action around the rich people tables or the poor ones. But we will not talk about the pleasures of good food. Instead, we are going to face he food need as an element that brings the necessary strength for one to stay alive. In this sense, we make a question: Like Primo Levi, in his book Se questo è un uomo, is there a man that eats a flavourless, colourless, oudorless food without any pleasure that serves just to make him keep alive? We will also see the playful and happy way Begnini uses in the beginning of his film La vita è bella to talk about food. But, in its second part, when his life is no more playful, although he leads it as if it still were so his son couldn't notice the place they had been conduct to, make all the possible efforts in order not to leave his son, Giosuè, without the necessary food.

KEYWORDS: literature; movies; concentration camp; food. 
1 cibo è l'elemento vitale dell'uomo, sia per il suo corpo che per la sua anima; ̀̀ il bagaglio culturale delle civiltà, ed è, quindi, capace di mostrare le caratteristiche delle varie società, facendole distinguere le une dalle altre. Perciò, il cibo è qualcosa di più che semplice materia utile al buon funzionamento del corpo, caricandosi di significati e simboli fondamentali nella vita di ogni persona.

Vito Teti, nel suo saggio Il colore del $c i b o^{2}$, distingue due modelli nutrizionali: quello ricco e quello povero: il rosso della carne che si contrappone al verde degli ortaggi e delle erbe, così come il pane bianco e quello nero. Polente, zuppe, minestre, carne essiccata e pane nero erano i cibi consumati dalla civiltà contadina, mentre cacciagione, frutta, pane bianco e spezie caratterizzavano quella nobiliare ${ }^{3}$. Il pane, specialmente nelle società mediterranee, è sempre stato considerato il cibo principale, assoluto, senza il quale era la carestia ${ }^{4}$.

La letteratura ha sempre riservato un ruolo di grande importanza al cibo ed

2. TETI, V., Il colore del cibo. Roma: Meltemi, 1999, cap. 5

3. MONTANARI, R. La fame e l'abbondanza: storia dell'alimentazione in Europa. Bari: Ed. Laterza, 1993, p.76-87

4. CAMPORESI, P. La terra e la luna: dai riti agrari ai fast food, un viaggio nel ventre dell'Italia. Milano: Garzanti, 1995, cap.1 
ai diversi valori culturali, umani e sociali che il preparare e consumare un cibo porta con sé. Non stupisce dunque che, dovuto al bisogno elementare che l'uomo ha del cibo (ed al suo trasformarsi in desiderio), molti artisti gli abbiano dedicato grande attenzione, così come avviene con il teatro, la pittura e soprattutto il cinema: quest'ultimo una forma d'arte in cui il bisogno e il desiderio di mangiare sono stati tematizzati e declinati in modi diversissimi.

Il legame tra il cibo e la letteratura è una cosa che, fin dai tempi più antichi, affascina tutti gli autori: i miti, le leggende, le fiabe ed i racconti popolari hanno sempre avuto nel cibo un elemento molto frequente. Negli ultimi tempi, la presenza del cibo nella narrativa è sempre maggiore, ed è, perciò, aumentato il numero di romanzi e racconti in cui il cibo assume un ruolo fondamentale, ed in alcuni casi è addirittura il protagonista assoluto: il cibo seduttivo nella letteratura di Grazia Deledda, il Manifesto della cucina futurista di Marinetti, gli scritti ricchi di riferimenti gastronomici e descrizioni culinarie di Carlo Emilio Gadda, la seduzione degli "intingoletti" di Mirandolina, le zuppe povere e calde nelle locande dei Promessi Sposi, il cibo da poveri dei vinti di Giovanni Verga, il ricordo dei pranzi della domenica che tengono in vita Primo Levi; l'odiosa zuppa di patate ed un pezzo di pane nero, che dovevano tenerlo vivo nel Lager, da dove voleva assolutamente uscire per dire al mondo le atrocità che lí succedevano.

Vorrei, quindi, parlare un po' sulla necessità del cibo come alimento che dá forza per continuare a vivere. Ed alla maniera di Primo Levi, domandiamo: è uomo chi mangia un cibo senza sapore, senza colore, senza odore e quindi senza piacere, che gli serve solo per vivere? È uomo chi vive dietro a dei fili spinati aspettando solo il momento della morte ed ogni sera si corica e dorme perché la stanchezza lo vince, perché dormendo può sognare e il sogno lo riporta a casa, dove c'è una tavola che, insieme alla compagnia dei cari parenti, offre cibi caldi e saporosi? E il sogno è sempre interrotto dal grido atroce della sveglia, Wstac: comincia un altro giorno, un giorno fatto di fame, umiliazioni, lavori forzati, degradazione, dolore... morte.

Questo è Primo Levi, l'uomo che ha vissuto come un non-uomo, insieme a molti altri visi anonimi di ebrei, che avevano perduto qualunque apparenza di uomo, insieme a quelli che, per sopravvivere e per usufrire di una vita un po' migliore erano diventati 
gli aguzzini dei propri fratelli giudei, avendo "venduto la loro anima al diavolo".

Che la fame e la ricerca del cibo hanno infatti dato origine a furti, litigi, trattative, sogni rievocazione, gesti abietti, imprese coraggiose e avventure di ogni tipo, è del tutto ovvio, nella situazione in cui Levi si era trovato. (GIGLIOLI, 1997, p. 400).

In Se questo è un uomo ${ }^{5}$, il libro in cui Levi narra le esperienze della deportazione nel Lager nazista di Buna-Monowitz nei pressi di Auschwitz, il cibo è l'oggetto di cui lui parla di più. Ma la forma come lo fa riesce a rendere interessante e principalmente ad essere buona conduttrice di scene di avventure tragiche, comiche, patetiche o picaresche.

Il cibo è evocato in una scena in cui Primo descrive dei volti familiari raccolti intorno ad una tavola e la possibilità di raccontare gli eventi, fuori da ogni norma di cui era stato insieme protagonista e testimone. Mangiare e raccontare: raccontare, in primo luogo, della fame, la più quotidiana delle infrazioni alla normalità che l'universo concentrazionario comportava, quella fame "sconosciuta agli uomini liberi, che fa sognare di notte" (LEVI, 1989, p. 31).

L'aspetto piú tragico della vita nel Lager non è costituito dalla lotta giornaliera per la sopravvivenza, cioè dalla capacità di resistere ai soprusi, alla fatica, alla mancanza di cibo, alle malattie, ma è costituito dalla degradazione dell'uomo, dal suo esser ridotto a zero, dal suo "vendersi" per un pezzo di pane o per una gamella di quella terribile zuppa, unico alimento, oltre al pane nero.

E vedremo ora, dai brani sotto, cosa significava il cibo in quell'inferno.

Levi, fin dall'inzio del suo voler scrivere per voler raccontare, anzi del suo voler relatare come testimone di quel grande misfatto, parla della fame e del cibo nella poesia epigrafe del libro (LEVI, 1989, p. 7)

[...] Voi che trovate tornando a sera

Il cibo caldo e visi amici

Considerate se questo è un uomo

[...] Che lotta per mezzo pane. 
Il tema cibo appare già quando le donne lo preparano con cura per i loro figli, che porteranno nel loro viaggio... un viaggio verso il nulla.

[...] Ma le madri vegliarono a preparare con dolce cura il cibo per il viaggio [...] Non fareste anche voi altrettanto? Se dovessero uccidervi domani col vostro bambino, voi non gli dareste oggi da mangiare?

(LEVI, 1989, p. 13)

Come dice Bruno Fronzi in L'uomo a zero:

[...] Già in queste righe traspare ciò che maggiormente focalizza l'interesse dell' autore, e che è adombrato nel titolo stesso del libro: la conservazione, pur di fronte alla morte imminente, di quella particolare qualità che l'uomo deve possedere per essere tale: 1"umanità'. Il Lager, la macchina che i tedeschi, con l'ingegno allucinato ma altamente raziocinante di certi alienati, hanno ideata, è volto appunto alla distruzione, prima che della fisica degli internati, della loro umanità. (FRONZI, 1997, p. 116).

Comincia cosí la degradazione dell'uomo, spogliato dei suoi abiti, capelli, nome, infine di tutto ciò che era suo, della sua cultura, della vita di uomo libero. Spogliati di ogni libertà e di ogni individualità, le vittime del Lager devono ridurre il proprio livello di esistenza al puro momento biologico: la fame e il cibo diventano la sola ragione d'esistenza. Non c'è più posto per la spiritualità, per l'interiorità, per le manifestazioni dell'arte o della cultura.

[...] Pare che questa sia l'iniziazione vera e propria: solo mostrando il numero si riceve il pane e la zuppa. (LEVI, 1989, p. 24),

[...] Abbiamo appreso il valore degli alimenti; ora anche noi raschiamo diligentemente il fondo della gamella dopo il rancio, e la teniamo sotto il mento quando mangiamo il pane per non disperderne le briciole. Anche noi adesso sappiamo che non è la stessa cosa ricevere il mestolo di zuppa prelevato dalla superficie o dal fondo del mastello, e siamo già in grado di calcolare, in base alla capacità dei vari mastelli, quale sia il posto più conveniente a cui aspirare quando ci si mette in coda. (LEVI, 1989 , p. 28 e 29). 
[...] Ho troppe cose da chiedere. Ho fame, e quando domani distribuiranno la zuppa, come farò a mangiarla senza cucchiaio? E come si può avere un chucchiaio? (LEVI, 1989, p. 33).

[...] Dopo quindici giorni dall'ingresso, già ho la fame regolamentare, la fame cronica sconosciuta agli uomini liberi, che fa sognare di notte e risiede in tutte le membra dei nostri corpi... (LEVI, 1989, p. 3.

Suona la sveglia: i prigionieri si vestono sommariamente, si lavano ed orinano correndo per risparmiare tempo perché inizierà la distribuzione del pane:

[...] del pane-Brot-Broit-chleb-pain-lechem-kenyér, del sacro blocchetto grigio... [...] Il pane è anche la nostra sola moneta... (LEVI, , p. 34).

Nel brano che segue, l'autore mostra come la degradazione causi, ormai, indifferenza a tutto, persino al cibo.

[...] Tutto gli è a tal segno indifferente che non si cura più di evitar la fatica e le percosse e di cercare il cibo. (LEVI, 1989, p. 38).

[...] Ora siamo pronti per la visita definitiva. Fuori dalla finestra si vede il cielo bianco, e qualche volta il sole; [...] A giudicare dalla sua posizione, debbono essere le quattordici passate: addio zuppa ormai, e siamo in piedi da dieci ore e nudi da sei. (LEVI, 1989, p. 42).

Un treno che passa fa sognare Primo:

[...] E passerebbe una donna, e mi chiederebbe [...] e io le racconterei [...] e lei capirebbe e mi darebbe da mangiare e da dormire. (LEVI, 1989, p. 38).

Ed un treno è ancora nei sogni di Primo, un treno che lo riporta a casa:

[...] Qui c'è mia sorella, e qualche mio amico non precisato, e molta altra 
gente. Tutti mi stanno ascoltando, e io sto raccontando proprio questo: [...] Racconto anche diffusamente nella nostra fame e... (LEVI, 1989, p. 53).

Nel Lager il cibo era considerato una moneta forte, una vera e propria borsa di valori: un pezzo di pane poteva far comprare vari oggetti, come zuppa, camicie, ecc, poi rivenduti ad un valore più alto:

[...] La Borsa è attivissima sempre. Ci sono stati periodi in cui per il buono-premio si è pagata una razione di pane, poi una e un quarto, anche una e un terzo; un giorno è stato quotato una razione e mezza... [...] Quest'ultimo si accontenterà di un modesto quantitativo di pane per accettare il cambio... (LEVI, 1989, p. 74).

Nel laboratorio chimico dove lui comincia a lavorare tutto è piú semplice:

[...] Si tratta di rubare o ricettare qualcuno degli svariati attrezzi, utensili [...] trovare il cliente ed effettuare il baratto conto pane e zuppa. (LEVI, 1989, p. 75) .

Primo Levi, nel brano che segue, descrive l'azione di compra-vendita degli infermieri del Ka-be:

[...] Gli infermieri poi traggono ingente guadagno dal traffico dei cucchiai. Il Lager non fornisce cucchiaio ai nuovi arrivati, benché la zuppa semiliquida non possa venir consumata altrimenti. (LEVI, 1989, p. 77).

[...] Occorre premettere che ogni baracca riceve, a sera, un'assegnazione di zuppa che è alquanto piú alta della somma delle razioni regolamentari. (LEVI, 1989, p. 76).

Qui Levi mostra come lo stare in Lager possa far diminuire moralmente le persone, ma se queste sono perseveranti riescono a sopravvivere:

[...] L. dirigeva nel suo paese una importantissima fabbrica. [..] in quel tempo i suoi privilegi si limitavano alla pulitura giornaliera della mar- 
mitta degli operai polacchi [...] questo lavoro, di cui egli aveva ottenuto non so come l'esclusività, gli fruttava mezza gamella di zuppa al giorno [...] L. se l'era saputa guadagnare con incredibile tenacia, pagando i singoli acquisti e servizi con pane della sua stessa razione, e astringendosi cosí a un regime di privazioni supplementari. (LEVI, 1989, p. 85).

Ed è nel 'Il canto di Ulisse' il capitolo più poetico, mentre Primo e Pikolo portano la zuppa, che quest'ultimo chiede a Primo di insegnargli l'italiano, e Primo usa alcuni versi della Divina Commedia e quel ritorno alla cultura lo fa sentire di nuovo un uomo degno.

[...] Darei la zuppa di oggi per sapere saldare "non ne avevo alcuna" col finale. (LEVI, 1989, p. 102).

[...] Siamo oramai nella fila per la zuppa, in mezzo alla folla sordida e sbrindellata dei porta-zuppa degli altri Kommandos [...] Si annunzia ufficialmente che oggi la zuppa è di cavoli e rape. (LEVI, 1989, p. 103).

Ed i loro rapporti con i civili non sono migliori di quelli con i tedeschi: sono da loro disprezzati e commiserati perché li considerano dei cenciosi, dei ladri, e affamati...

[...] Naturalmente questo non impedisce a molti di loro di gettarci qualche volta un pezzo di pane o una patata, o di affidarci, dopo la distribuzione della Zivilsuppe in cantiere, le loro gamelle da raschiare e restituire lavate. (LEVI, 1989, p. 108).

Nell'ottobre del 1944 giá molti di loro erano morti. Ora ci sarebbe stata una nuova selezione, alla quale Primo riesce a scappare:

[...] Nella nostra baracca la selezione è ormai finita, però continua nelle altre, [...] ma poiché frattanto i bidoni della zuppa sono arrivati... Ai selezionati verrà distribuita doppia razione [di zuppa]. Non ho mai saputo se questa fosse un'iniziativa assurdamente pietosa .......] Ziegler presenta la gamella, riscuote la normale razione, poi resta lí in attesa. [...] ha diritto alla doppia razione. Quando l'ha ottenuta se ne va in cuccetta a 
mangiare. (LEVI, 1989, p. 115 e 116).

Ma il raccontare del cibo è molto forte specialmente nell'ultimo capitolo, perché è il cibo che aiuterà quei pochi relitti, lasciati dai tedeschi nell'infermeria, a restar vivi, e Primo, facendo un diario ancora piú dettagliato di quei dieci giorni, relata le loro ricerche per trovare le patate, principale cibo di quel momento.

[...] Ci dirigemmo alle cucine piú in fretta che potemmo, ma le patate erano già quasi finite. [...] La legge del Lager diceva: "Mangia il tuo pane, e, se puoi, quello del tuo vicino" e non lasciava posto per la gratitudine. (LEVI, 1989:141-142)

[...] Ci restarono viveri (e cioè patate) per due giorni soltanto: per l'acqua eravamo ridotti a fondere la neve, operazione penosa per la mancanza di grandi recipienti, da cui si otteneva un liquido marastro e torbido che era necessario filtrare. [...] Nella cucina, due si accapigliavano per le ultime decine di patate putride...[...] Nel cortile del magazzino stavano due grandi mucchi di cavoli e di rape (le grosse rape insipide, base della nostra alimentazione). (LEVI, 1989, p. 143).

[...] Per quel giorno ci accontentammo ancora di patate bollite e fette di rapa arrostite sulla stufa... ( LEVI, 1989, p. 144).

[...] Dovevamo inaugurare i cavoli e le rape.[...] La notizia che una zuppa era in cottura si sparse rapidamente fra la folla dei semivivi; si formò sulla porta un assembramento di visi famelici. (LEVI, 1989, p. 145).

[...] I piú si dispersero, ma uno si fece avanti...[...] In cambio di un litro di zuppa si sarebbe messo a nostra disposizione per tagliarci abiti dalle numerose coperte rimaste in campo. [...] A sera, dopo la prima zuppa distribuita con entusiamo e divorata con avidità il grande silenzio della pianura fu rotto... (LEVI, 1989, p. 146).

Più tardi, Primo sveglia chi già stava dormendo e parla a tutti sulla necessità di badare alle proprie cose, perché ormai le malattie infettive si erano propagate con intensità: 
[...] Che ognuno conservasse attentamente la sua propria gamella e il cucchiaio, che nessuno offrisse ad altri la zuppa che gli fosse avanzata... (LEVI, 1989, p. 146).

Il giorno dopo, Primo ed un suo compagno vanno fino al campo delle SS, fuori dal reticolato. Primo quella sera si spinge fino al reparto di dissinterici per portare un po' di ristoro a due italiani:

[...] A sera quando tutti i lavori furono finiti vincendo la fatica e il ribrezzo, mi trascinai a tentoni per il corridoio lercio e buio, fino al loro reparato, con una gamella d'acqua e gli avanzi della nostra zuppa del giorno. (LEVI, 1989, p. 146).

E qui racconta il caso di un giovane il cui stato era cosi grave che la zuppa gli fece male:

[...] Forse causa di tutto fu la minestra di cavoli e rape, di cui Lakmaker aveva voluto due razioni. A metà notte gemette, poi si buttò giú da letto. Cercava di raggiungere la latrina .... affinché il tifo non si spargesse [...] Le nostre patate erano finite. Circolava da giorni per le baracche la voce che un enorme silo di patate fosse situato da qualche parte, fuori dal filo spinato, non lontano dal campo... (LEVI, 1989, p. 148).

Ed è lí che Primo ed un suo compagno vanno a cercare del cibo e, per la prima volta dopo l'arresto, si sentono liberi.

[...] A forse quattrocento metri dal campo giacevano le patate: un tesoro. Due fosse lunghissime piene di patate. [... ] Nessuno sarebbe piú morto di fame, la estrazione era molto difficile perché era tutto congelato. [...] Un vecchio ungherese era stato sorpreso colà dalla morte. Giaceva irrigidito nell'atto dell'affamato [...] Da allora il nostro vitto migliorò. Oltre alle patate bollite e alla zuppa di patate, offrimmo ai nostri malati frittelle di patate... (LEVI, 1989, p. 149).

[...] Ma nella baracca 14 vi erano ricoverati gli operai che organizzarono 
una spedizione al campo degli inglesi e ne ritornarono con un carretto pieno di "meraviglie mai viste: margarina, polveri per budino, lardo, farina di soia, acquavite". ( LEVI, 1989, p. 149).

[...] Un convalescente di tifo e di scarlattina fu preso da una forte febbre e rimase 5 giorni zitto fino a quando disse: - Ho una razione di pane sotto il saccone. Dividetevela voi. Io non mangerò piú. (LEVI, 1989, p. 151).

I malati rimarranno nel Lager ormai devastato, senza cure, senz'acqua, senza cibo, decimati dal freddo, dal tifo, dalla difterite, dalla dissenteria... Cosí li troveranno i russi: pochi sopravvisuti devastati nel físico e nell'animo.

E concludiamo la prima parte di questo nostro lavoro riportando le parole amare dette da Primo per il fatto di non sentirsi più un uomo:

[...] È uomo chi uccide, è uomo chi fa o subisce un'ingiustizia, non è uomo chi ha perso ogni ritegno, divide il letto con un cadavere. Chi non ha atteso che il suo vicino finisse di morire per togliergli un quarto di pane, è, pur senza colpa, più lontano dal modello dell'uomo pensante... (LEVI, 1989, p. 152).

Invece in La vita è bella - il filme che mostra l'olocausto in maniera completamente differente dall'infinità di film il cui tema è la più grande tragedia umana del XX secolo - non ci è mostrata la fame ed il cibo come una delle maggiori sofferenze dell'uomo nel Lager perché Benigni non voleva fare un filme sulla degradazione e morte dell'uomo ebreo, ma voleva, come lui stesso diceva, raccontare una storia d'amore: amore per la bella sognatrice Dora e amore per il figlioletto che pagherà con il Lager l'eredità ebrea.

La prima volta il cibo ci appare sotto forma di uova che l'astuto Guido mette, alla maniera di Charles Chaplin, dentro il cappello.

Poi il cibo è arnese di lavoro: è il cameriere Guido, il quale per mostrare allo zio come servirà i clienti dell'hotel, inscena di tagliare e servire un bel pollo ed una non meno bella aragosta, che Benigni ci mostra in scene divertentissime. 
ZIO: Il pollo!

GUIDO: Facile! Il pollo si serve intero con il dorso sul piatto. "Me lo taglia!" "Prego!" Prima cosa tengo il pollo fermo con la lama del coltello infilzata sotto l'ala e stacco la coscia. Poi incido la carne lungo la carena dello sterno... Via ali e petto, via la pelle...

[...] ZIO: Aragosta!

Un lampo di panico s'accende negli occhi dell'apprendista cameriere...

GUIDO: Infilzo la pelle sotto l'ala... L'aragosta è un crostaceo. Via la crosta... (esita) via le antenne! L'aragosta l'abbiamo finita ... però abbiamo il pollo, vuole il pollo? (BENIGNI, 2002, p. 25).

Subito dopo, anche per farsi perdonare dallo zio lo sbaglio fatto, con il tovagliolo sul braccio comincia a mostrargli di essere un buon cameriere, apparecchiando una tavola i cui elementi hanno nomi stranissimi e servendo possibili ospiti, in scene ilarianti che strappano grandi risate agli spettatori.

Poi Benigni ci mostra la furbizia di Guido, quando nell'hotel porta un vassoio con salmone, insalata e un bicchiere di vino bianco, al tavolo di un capitano tedesco a cui piacevano gli indovinelli e che Guido aiuta a risolvere i piú difficili. Questi, preoccupato con la risposta dell'indovinello, non mangia e ritorna in camera dove si prepara per tornare in Germania, da dove era stato richiamato. Poco più tardi Guido deve servire la cena all'Ispettore della Pubblica Istruzione, appena arrivato da Roma, che vorrebbe mangiare "qualcosa di molto leggero". Guido immediatamente pensa alla cena in cui il capitano tedesco non aveva mangiato e decide di farla "scegliere" all'ispettore dandogli due alternative: una con dei cibi grassi e bisunti e l'altra con i cibi (magri) non mangiati dal capitano tedesco. È chiaro che l'ispettore finisce per scegliere esattamente quello che Guido voleva dargli, anche perché era stanco ed il cibo propostogli è già lí!

Più tardi appare una tavola imbandita per festeggiare il fidanzamento ufficiale di Dora, di cui Guido è innamorato, con un burocrata dalle idee fasciste. 
Lo zio, insieme a un altro cameriere, Ernesto, sta mettendo a punto la scenografia esotica di una grandissima torta "etiope", con tanto di laghetti, alberelli, montagnole, tucul, ecc. In mezzo alla torta, larga quasi un metro e mezzo di diametro, campeggia un piccolo struzzo finto con il becco spalancato sollevato verso l'alto. Arriva Guido con l'uovo nelle mani e lo porge bruscamente allo zio. [...] Il vecchio capocameriere lo prende delicatametne e lo incastra bene nel becco dello struzzo.

GUIDO: Ma è un dolce africano?

ZIO: Sí, è l'ultimo grido della pasticceria, la torta etiope, tutta fatta con zabaione di uova di struzzo. (Con tono ironico) Questo è l'Impero! La offriamo noi dell'albergo ai festeggiati. (BENIGNI, 2002, p. 33).

Davanti a quei cibi bellissmi da vedere e da gustare gl'invitati parlano delle ultime notizie sugli ebrei, sugli zingari e sui gay e quanto eliminarli costerebbe molto meno al governo italiano che il lasciarli vivere in libertà.

Poi Dora, che non amava il burocrata ed era stata obbligata dalla madre ad accettarlo, si lascia scivolare sotto quel tavolo pieno di cibi squisitissimi, dove "incontra" Guido: i due giovani si baciano e Dora chiede a Guido di portarla via. Attraversano il salone sulla groppa del cavallo dello zio, su cui proprio quella sera era stato scritto "cavallo ebreo", e che porterà i due giovani verso la... felicità.

Qui la scena è interrotta ed è ripresa alcuni anni dopo... Benigni ora ci mostra il trascorrere della vita di Dora e Guido e del loro piccolo Giosué. Il giorno del compleanno del bambino, Dora va a prendere la madre, che non vede piú dal momento dalla fuga del suo non-fidanzamento ufficiale con il burocrata; Guido e Giosué rimangono a casa e preparano la cena. Lo zio gli porta le stoviglie e della roba da mangiare, presa in hotel:

Su un piatto è posato uno strano volatile arrosto.

GUIDO: Ah, gallina...

ZIO: $\mathrm{Si}$, ma con una coscia sola. Le ali sono di piccione... non garantisco niente, tutti avanzi. Però faremo la nostra figura. (BENIGNI, 2002, p. 113). 
Quando Dora ritorna non li trova... Guido ed il figlio sono stati portati in un Lager tedesco e, affinché Giosuè non percepisca l'orrore della cosa, il padre gli fa credere che sono lì per partecipare ad un gioco. È chiaro che quel luogo non piace assolutamente al bambino che ha sonno e fame e chiede se avranno la merenda, al che il padre, preso di sorpresa, finge di domandare ai colleghi se "...è già passato quello che dà il pane con la marmellata" (BENIGNI, 2002, p. 120). E Guido coglie l'occasione per convincere il figlio che non può chiedere da mangiare altrimenti saranno sclassificati dal gioco. $\mathrm{E}$ glielo dice anche durante la strana traduzione dando degli ordini, diretti ai prigionieri, dettatigli dal soldato tedesco, che Guido traduce a modo suo per poter "comunicare" al figlio le leggi del gioco ed il bambino crede ciecamente alle parole del padre.

GUIDO: (mostra tre dita) In tre casi si perdono tutti i punti. Li perdono ...: uno quelli che si mettono a piangere. Due: quello che vogliono vedere la mamma. Tre: quelli che hanno fame e vogliono la merendina. (Forte)Scordatevela! (BENIGNI, 2002, p. 131).

Poi Guido, è invitato dal capitano tedesco, che si ospitava nell'hotel dello zio e che aveva incontrato durante una "selezione" nel Lager, a servire una cena agli ufficiali tedeschi. Il piccolo Giosuè, che era insieme al padre in uno spiazzo, è portato via da una soldatessa tedesca che sta badando alla cena dei figli degl'invitati. Prima di esser portato via, Guido fa in tempo a dire al bambino che non deve parlare per non far capire chi è. Poi Guido finge di insegnare a tutti i bambini a dire "Grazie" in italiano, perché Giosuè, da bambino educato com'era, se lo era fatto scappare. Lí il piccolo può finalmente mangiare bene:

I ragazzini stanno mangiando il dolce, sempre zitti. Giosuè ha la bocca stracolma, non ce la fa ad ingoiare. Il padre, con una scusa, si china alla sue spalle.

GUIDO (sussurra) Piano Giosuè, mangia piano che ti fa male. Siamo in testa, forse finisce prima il gioco. Ce ne andiamo prima, Giosuè! (BENIGNI, 2002, p. 170). 
Queste parole vogliono dimostrare che Guido aveva capito che la loro prigionia sarebbe finita in brevissimo tempo perché stavano per arrivare gli alleati,

ed i tedeschi avrebbero abbandonato il campo portandosi i prigionieri.

Subito dopo troviamo i due che si preparano a fuggire prima che siano portati via dai tedeschi in fuga, però vedono un camion che porta via delle donne e Guido tenta di farsi passare per una donna: si butta sul capo la giacca di Giosué e sul corpo la coperta per cercare fra di esse sua moglie: non la trova. Due soldati lo vedono e lo portano al 'solito posto', dove sarà subito ucciso. Anche in quel momento estremo la sua preoccupazione è Giosué, cioè che il piccolo non capisca dove lo stanno portando, così che cammina, fra i due tedeschi, come un burattino e fa l'occhiolino al figlio che aveva lasciato nascosto in un casotto.

E Guido, vestito da donna, e con l'aspetto di un burattino, esce di scena...

Giosuè l'indomani è salvato dagli inglesi che arrivano su di un carro armato, l'oggetto che il padre gli aveva fatto credere che avrebbero vinto alla fine del gioco. Subito dopo esser usciti dal Lager, Giosué vede la madre, seduta sul ciglio della strada a cui grida esultante: "Abbiamo vinto!"

\section{Conclusione}

Il cibo già nell'età antica aveva una struttura completa e varia ed era magnificato nei banchetti, nelle arti e nelle lettere. Con il Medioevo, la forte caduta sociale e culturale contagia anche il cibo. Le diversità anche a tavola sono ben note: mentre i ricchi avevano cibo a volontà, i poveri pativano fame perpetua. Nel Rinascimento, il cibo si arricchisce con nuovi prodotti agricoli provenienti dalle Americhe ed è sinonimo di potenza, di ostentazione di un'esagerata potenza commerciale, economica e finanziaria. Oggigiorno il cibo è fonte di una raffinata ricerca: ora i consumatori chiedono sempre di più piatti gustosi ed innovativi.

Come abbiamo visto nelle due opere in analisi, il cibo è l'elemento necessario alla vita dell'uomo, anche quando non mangia per piacere e gusto: è con il cibo che acquista la forza e l'energia necessarie alla sua sopravvivenza e, molte volte, finisce per degradarsi per ottenerlo, come avviene in Se questo è un uomo, anche perché l'uomo nel Lager è in stato di degradazione assoluta. E Primo Levi ne parla durante tutto il suo 
racconto, perciò le nostre citazioni sono varie.

Nel film La vita è bella, invece, Benigni "parla" del cibo in maniera più leggera. Inizialmente é come un motivo per far ridere un po', poi, anche qui, diventa necessità: una necessità di cui il piccolo Giosuè sente la mancanza e che il padre s'ingegna come può affinché non gli manchi e non gli manchi la certezza di poterlo avere. E ce lo mostra così anche quando nel Lager il bambino ha fame, perché in tutto il film lo stare in "quel lougo" è un fatto che non deve spaventarlo, anzi deve dargli allegria perché alla fine del loro "soggiorno" lí, cioè alla fine del gioco, potranno vincere un carroarmato in premio.

Abbiamo brevemente parlato su due opere infinite: infinite perché possono essere osservate da vari angoli, e noi ne abbiamo qui visto appena quello che parla sul cibo, cioè, quel cibo che per Primo Levi è piú che mai necessario al fisico ed alla mente dei prigionieri, che, ormai senza forze, senza nome, senza più dignità, non hanno più l'apparenza di uomini: quel misero cibo servirà a non far "morire" quelle persone che ormai degli uomini vivi ne hanno solo la fame. Mentre Benigni deve ingegnarsi, facendo del cibo un momento di ilarità affinché il piccolo non senta molto l'orrore di quel luogo e continui a pensare che tutto quello è un gioco.

\section{Riferimenti bibliografici}

BELPOLITI, M. (org.). Primo Levi: conversazioni ed interviste 1963-1987. Torino: Giulio Einaudi Editore, 1997.

(org.). Opere. Vol. I e II. Introduzione di Daniele Del Giudice. Torino: Giulio Einaudi Editore, 1997, pp. 343-45. (org.). Riga 13-Primo Levi. Milano: Marcos y Marcos, 1991.

BENIGNI, R. e CERAMI, V. La vita è bella. Torino: Giulio Einaudi Editore, 2002.

BIANCHINI, E. Invito alla lettura di Primo Levi. Milano: Gruppo Ugo Mursia Editore , 2000 .

BORSATTI, C. Roberto Benigni. Milano: Editrice Il Castoro, 2001.

BRAMBILLA, R. e CACCIATORE G. (org.). Primo Levi: la dignità dell'uomo. Assisi: Cittadella Editrice, 1995.

CALCAGNO, G. Primo Levi: capire non è perdonare. In: Tuttolibri, 26/07/1987.

DALLA BONA, F. Literatura e gastronomia: um casamento perfeito. São Paulo: Itália Nova Editora, 2005. 
DINI, M. e JESURUM S. Primo Levi: le opere e i giorni. Milano: Rizzoli Libri, 1992.

FRONZI, B. L'uomo a zero. In Riga 13-Primo Levi (a cura di Marco Belpoliti), Milano: Marcos y Marcos,1997.

GIGLIOLI, D. Narratore. In Riga 13-Primo Levi (a cura di Marco Belpoliti), Milano: Marcos y Marcos, 1997.

LEVI, Primo. Se questo è un uomo-La tregua.Torino: Giulio Einaudi Editrice, 1989.

MATTIODA, E. (org). Al di qua del bene e del male - La visione del mondo di Primo Levi. Atti del convegno internazionale. Torino 15-16 dic. 1999. Milano: F. Angeli, 2000.

MOLITERNI, F. et alii. Primo Levi: l'a-topia letteraria; il pensiero narrativo; la scrittura e l'assurdo. Napoli: Liguori, 2000.

MOMIGLIANO, P. e GORRIS R. (org.). Primo Levi: testimone e scrittore di storia. Firenze: Editrice La Giuntina, 1999.

PAULETTO, P. In Riga 13. Milano: Marcos y Marcos, 1997.

POLI, G., CALCAGNO G.. Echi di una voce perduta: incontri, interviste e conversazioni con Primo Levi. Milano: Gruppo Ugo Mursia Editore, 1992.

SEGRE, C. Lettura di 'Se questo è un uomo'. In: Primo Levi: Un'antologia della critica - a cura di E. Ferrero. Torino: Piccola Biblioteca Einaudi, 1997.

SPADI, M. Le parole di un uomo - Incontro con Primo Levi. Roma: Di Renzo Editore, 1997. TOSCANI, C. Come leggere 'Se questo è un uomo'. Milano: Mursia, 1990.

VINCENTI, F. Invito alla lettura di Primo Levi. Milano: Mursia, 1973. 